\title{
Inhomogeneous Equation of State of the Universe: Phantom Era, Future Singularity and Crossing the Phantom Barrier
}

\author{
Shin'ichi Nojiri' ${ }^{1}$ and Sergei D. Odintsov ${ }^{2}$ \\ ${ }^{1}$ Department of Applied Physics, National Defence Academy, Hashirimizu Yokosuka 239-8686, Japan \\ ${ }^{2}$ Instituciò Catalana de Recerca i Estudis Avançats (ICREA) and Institut d'Estudis Espacials de Catalunya (IEEC), \\ Edifici Nexus, Gran Capità 2-4, 08034 Barcelona, Spain
}

(Dated: September 26, 2018)

\begin{abstract}
The dark energy universe equation of state (EOS) with inhomogeneous, Hubble parameter dependent term is considered. The motivation to introduce such a term comes from time-dependent viscosity considerations and modifications of general relativity. For several explicit examples of such EOS it is demonstrated how the type of future singularity changes, how the phantom epoch emerges and how crossing of phantom barrier occurs. Similar cosmological regimes are considered for the universe with two interacting fluids and for universe with implicit EOS. For instance, the crossing of phantom barrier is realized in easier way, thanks to the presence of inhomogeneous term. The thermodynamical dark energy model is presented where the universe entropy may be positive even at phantom era as a result of crossing of $w=-1$ barrier.
\end{abstract}

PACS numbers: $98.70 . \mathrm{Vc}$

\section{INTRODUCTION}

The increasing number of evidences from the observational data indicates that current universe lives in a narrow strip near $w=-1$ (where $\mathrm{w}$ is the equation of state (EOS) parameter), quite probably being below -1 in socalled phantom region. It is also assumed that modern universe is filled with some mysterious, negative pressure fluid (dark energy) which represents about 70 percents of total energy in the universe. (The simplest, phenomenological approach is to consider that this fluid satisfies to EOS with constant $w$ ). The origin of this dark energy is really dark: the proposed explanations vary from the modifications of gravity to the introduction of new fields (scalars, spinors, etc) with really strange properties. Moreover, forgetting for the moment about the origin of dark energy, even more-less satisfactory mechanism of evolving dark energy is missing, so far. At best, each of existing theoretical models for dark energy explains some specific element(s) of late-time evolution, lacking the complete understanding. Definitely, the situation may be improved with the new generation of observational data when they will present the realistic evolving EOS of dark energy during sufficiently large period.

The most strange (if realistic) era in the universe evolution is phantom era. There are many attempts to describe the phantom cosmology (see, for instance, 1, 2] and references therein), especially near to future, finitetime singularity (Big Rip) which is the essential element of classical phantom cosmology. (Note that quantum effects may basically provide the escape from future, finite type singularity, for recent discussion, see [3, 4]). Unfortunately, the easiest way to describe the phantom cosmology in the Lagrangian formulation leads to the necessity of the introduction of not very appreciated scalar with negative kinetic energy [5]. Another, easy way is to use some phenomenological EOS which may produce dark epoch of the universe (whatever it is). It is re- markable that such description shows the possibility of other types of future, finite type singularity. For instance, even when EOS is suddenly phantomic (near to rip time where negative pressure diverges), the sudden singularity occurs [6]. There may exist future singularities where energy/pressure is finite at rip time, for classification of future singularities, see [4]. They may occur even in modified gravity at late times, see 7] for explicit examples. Nevertheless, it is remarkable that effective phantom phase may be produced also in string-inspired gravities [8].

The present paper is devoted to study the phantom cosmology and related regimes (for instance, crossing of phantom divide) when phenomenological equation of state of the universe is inhomogeneous. In other words, it contains terms dependent explicitly from Hubble parameter (or, even from its derivatives). Definitely, one needs quite strong motivation for such modification of dark energy EOS. The first one comes from the consideration of time-dependent bulk viscosity 9, 10]. (For earlier discussion of cosmology with time-dependent bulk viscosity, see see also 11].) Actually, it was constructed the specific model of dark energy with possibility of crossing of phantom divide due to time-dependent bulk viscosity [9]. The construction of EOS from symmetry considerations 12 indicates to the necessity of some inhomogeneous correction. Finally, big number of gravities: from low-energy string effective actions to gravity with higher derivative terms or with inverse terms on curvatures modifies the FRW equations in requested form.

The paper is organized as follows. In the next section we consider spatially-flat FRW universe filled by the ideal fluid with specific, dark energy EOS 3]. Short review of four types of future singularity for different choices of EOS parameters is given, following to ref. [4]. The inhomogeneous term of specific form is introduced to EOS. The role of such term in the transition of different types of singularity to another ones is investigated. 
The cosmological regimes crossing phantom barrier due to such terms are explicitly constructed. Finally, the dependence of the inhomogeneous term from Hubble parameter derivatives is briefly discussed as well as emerging oscillating universe. Section three is devoted to the study of similar questions when FRW universe is filled by the interacting mixture of two fluids. The modification of two fluids EOS by inhomogeneous term is again considered. The explicit example of late-time cosmology (which may be oscillating one) quite naturally crossing the phantom divide in such a universe is presented. It is interesting that inhomogeneous term may effectively compensate the interaction between two fluids. In the section four we discuss the FRW cosmology admitting the crossing of barrier $w=-1$ due to specific form of the implicit dark energy EOS proposed in ref. 13]. Again, the generalized, Hubble parameter dependent EOS is considered. Some thermodynamical dark energy model passing the barrier $w=-1$ is constructed, based on above EOS. It is demonstrated that in such a model the universe entropy may be positive even during the phantom era. Some summary and outlook are given in the discussion section. The Appendix deals with couple simple versions of modified gravity which may predict the requested generalization of EOS.

\section{FRW COSMOLOGY WITH INHOMOGENEOUS DARK ENERGY EQUATION OF STATE}

In the present section we make brief review of FRW cosmology with explicit dark energy equation of state (power law). The modification of EOS by Hubble dependent term (constrained by energy conservation law) is done and its role to FRW cosmology evolution is investigated. The starting FRW universe metric is:

$$
d s^{2}=-d t^{2}+a(t)^{2} \sum_{i=1}^{3}\left(d x^{i}\right)^{2} .
$$

In the FRW universe, the energy conservation law can be expressed as

$$
0=\dot{\rho}+3 H(p+\rho) .
$$

Here $\rho$ is energy density, $p$ is pressure. The Hubble rate $H$ is defined by $H \equiv \dot{a} / a$. When $\rho$ and $p$ satisfy the following simple EOS:

$$
p=w \rho,
$$

and if $w$ is a constant, Eq.(2) can be easily integrated:

$$
\rho=\rho_{0} a^{-3(1+w)} .
$$

Using the first FRW equation

$$
\frac{3}{\kappa^{2}} H^{2}=\rho
$$

the well-known solution follows

$$
a=a_{0}\left(t-t_{1}\right)^{\frac{2}{3(w+1)}} \quad \text { or } \quad a_{0}\left(t_{2}-t\right)^{\frac{2}{3(w+1)}},
$$

when $w \neq-1$, and

$$
a=a_{0} \mathrm{e}^{\kappa t \sqrt{\frac{\rho_{0}}{3}}}
$$

when $w=-1$. In ([6), $t_{1}$ and $t_{2}$ are constants of the integration. Eq. (7) expresses the deSitter universe. In (6), since the exponent $2 / 3(w+1)$ is not integer in general, we find $t>t_{1}$ or $t<t_{2}$ so that $a$ should be real number. If the exponent $2 / 3(w+1)$ is positive, the first solution in (6) expresses the expanding universe but the second one expresses the shrinking universe. If the exponent $2 / 3(w+1)$ is negative, the first solution in (6) expresses the shrinking universe but the second one expresses the expanding universe. In the following, we only consider the case that the universe is expanding. Then for the second solution, however, there appears a singularity in a finite time at $t=t_{2}$, which is called the Big Rip singularity ( for discussion of phantom cosmology near Big Rip and related questions, see [1, 2] and references therein) when

$$
w<-1
$$

In general, the singularities may behave in different ways. One may classify the future singularities as following [4]:

- Type I ("Big Rip") : For $t \rightarrow t_{s}, a \rightarrow \infty, \rho \rightarrow \infty$ and $|p| \rightarrow \infty$

- Type II ("sudden") : For $t \rightarrow t_{s}, a \rightarrow a_{s}, \rho \rightarrow \rho_{s}$ or 0 and $|p| \rightarrow \infty$

- Type III : For $t \rightarrow t_{s}, a \rightarrow a_{s}, \rho \rightarrow \infty$ and $|p| \rightarrow \infty$

- Type IV : For $t \rightarrow t_{s}, a \rightarrow a_{s}, \rho \rightarrow 0,|p| \rightarrow 0$ and higher derivatives of $H$ diverge. This also includes the case when $\rho(p)$ or both of them tend to some finite values while higher derivatives of $H$ diverge.

Here $t_{s}, a_{s}$ and $\rho_{s}$ are constants with $a_{s} \neq 0$. The type I may correspond to the Big Rip singularity [1], which emerges when $w<-1$ in (3). The type II corresponds to the sudden future singularity [6] at which $a$ and $\rho$ are finite but $p$ diverges. The type III appears for the model with $p=-\rho-A \rho^{\alpha}$ [14], which is different from the sudden future singularity in the sense that $\rho$ diverges. This type of singularity has been discovered in the model of Ref. [3] where the corresponding Lagrangian model of a scalar field with potential has been constructed.

One may start from the dark energy EOS as

$$
p=-\rho-f(\rho),
$$

where $f(\rho)$ can be an arbitrary function in general. The function $f(\rho) \propto \rho^{\alpha}$ with a constant $\alpha$ was proposed in 
Ref. [3] and was investigated in detail in Ref. [14]. Using (2) for such choice, the scale factor is given by

$$
a=a_{0} \exp \left(\frac{1}{3} \int \frac{d \rho}{f(\rho)}\right) .
$$

Using (5) the cosmological time may be found

$$
t=\int \frac{d \rho}{\kappa \sqrt{3 \rho} f(\rho)},
$$

In case

$$
f(\rho)=A \rho^{\alpha},
$$

by using Eq. (10), it follows

$$
a=a_{0} \exp \left[\frac{\rho^{1-\alpha}}{3(1-\alpha) A}\right] .
$$

When $\alpha>1$, the scale factor remains finite even if $\rho$ goes to infinity. When $\alpha<1, a \rightarrow \infty(a \rightarrow 0)$ as $\rho \rightarrow \infty$ for $A>0(A<0)$. Since the pressure is now given by

$$
p=-\rho-A \rho^{\alpha},
$$

$p$ always diverges when $\rho$ becomes infinite. If $\alpha>1$, the EOS parameter $w=p / \rho$ also goes to infinity, that is, $w \rightarrow+\infty(-\infty)$ for $A<0(A>0)$. When $\alpha<1$, we have $w \rightarrow-1+0(-1-0)$ for $A<0(A>0)$ as $\rho \rightarrow \infty$.

By using Eq.(11) for (12), one finds [4]

$$
t=t_{0}+\frac{2}{\sqrt{3} \kappa A} \frac{\rho^{-\alpha+1 / 2}}{1-2 \alpha}, \quad \text { for } \quad \alpha \neq \frac{1}{2},
$$

and

$$
t=t_{0}+\frac{\ln \left(\frac{\rho}{\rho_{0}}\right)}{\sqrt{3} \kappa A}, \quad \text { for } \quad \alpha=\frac{1}{2} .
$$

Therefore if $\alpha \leq 1 / 2, \rho$ diverges in an infinite future or past. On the other hand, if $\alpha>1 / 2$, the divergence of $\rho$ corresponds to a finite future or past. In case of finite future, the singularity could be regarded as a Big Rip or type I singularity. 4]:

For the choice (12), the following cases were discussed

- In case $\alpha=1 / 2$ or $\alpha=0$, there does not appear any singularity.

- In case $\alpha>1$, Eq. 15 tells that when $t \rightarrow t_{0}$, the energy density behaves as $\rho \rightarrow \infty$ and therefore $|p| \rightarrow \infty$ due to (14). Eq.(13) shows that the scale factor $a$ is finite even if $\rho \rightarrow \infty$. Therefore $\alpha>1$ case corresponds to type III singularity.

- $\alpha=1$ case corresponds to the case (B) if we replace $-1-A$ with $w$. Therefore if $A>0$, there occurs the Big Rip or type I singularity but if $A \leq 0$, there does not appear future singularity.
- In case $1 / 2<\alpha<1$, when $t \rightarrow t_{0}$, all of $\rho,|p|$, and $a$ diverge if $A>0$ then this corresponds to type I singularity.

- In case $0<\alpha<1 / 2$, when $t \rightarrow t_{0}$, we find $\rho$, $|p| \rightarrow 0$ and $a \rightarrow a_{0}$ but by combining (13) and (15), we find

$$
\ln a \sim\left|t-t_{0}\right|^{\frac{\alpha-1}{\alpha-1 / 2}}
$$

Since the exponent $(\alpha-1) /(\alpha-1 / 2)$ is not always an integer, even if $a$ is finite, the higher derivatives of $H$ diverge in general. Therefore this case corresponds to type IV singularity.

- In case $\alpha<0$, when $t \rightarrow t_{0}$, we find $\rho \rightarrow 0, a \rightarrow a_{0}$ but $|p| \rightarrow \infty$. Therefore this case corresponds to type II singularity.

Hence, the brief review of FRW cosmology with specific homogeneous EOS as well as its late-time behaviour (singularities) is given (see [4] for more detail).

At the next step, we will consider the inhomogeneous EOS for dark energy, so that the dependence from Hubble parameter is included in EOS. The motivation for such EOS comes from including of time-dependent bulk viscosity in ideal fluid EOS [9] or from the modification of gravity (see Appendix). Hence, we suggest the following EOS

$$
p=-\rho+f(\rho)+G(H) \text {. }
$$

where $G(H)$ is some function. Then the energy conservation law (2) has the following form:

$$
0=\dot{\rho}+3 H(f(\rho)+G(H)) .
$$

By using the first FRW equation (5) and assuming the expanding universe $(H \geq 0)$, one finds

$$
\dot{\rho}=F(\rho) \equiv-3 \kappa \sqrt{\frac{\rho}{3}}(f(\rho)+G(\kappa \sqrt{\rho / 3})) .
$$

or

$$
G(H)=-f\left(3 H^{2} / \kappa^{2}\right)+\frac{2}{\kappa^{2}} \dot{H} .
$$

Hence, one can express $G(H)$ in terms of $f$ as above.

As a first example, let assume that EOS(3) could be modified as

$$
p=w_{0} \rho+w_{1} H^{2} .
$$

Using (5), it follows

$$
p=\left(w_{0}+\frac{\kappa^{2} w_{1}}{3}\right) \rho .
$$

Therefore $w$ is effectively shifted as

$$
w \rightarrow w_{\text {eff }} \equiv w_{0}+\frac{\kappa^{2} w_{1}}{3} .
$$


Then even if $w_{0}<-1$, as long as $w_{\text {eff }}>-1$, there does not occur the Big Rip singularity. From another side one can start with quintessence value of $w_{0}$, the inhomogeneous EOS (23) with sufficiently negative $w_{1}$ brings the cosmology to phantom era.

As a second example, we assume $f(\rho)$ (12) is modified as

$$
f(\rho)=A \rho^{\alpha} \rightarrow f(\rho)+G(H)=-A \rho^{\alpha}-B H^{2 \beta} .
$$

By using the first FRW equation (15), we find $f(\rho)$ is modified as

$$
\begin{aligned}
& f_{\mathrm{eff}}(\rho)=f(\rho)+G(H)=-A \rho^{\alpha}-B^{\prime} \rho^{\beta}, \\
& B^{\prime} \equiv B\left(\frac{\kappa^{2}}{3}\right)^{\beta} .
\end{aligned}
$$

If $\beta>\alpha$, when $\rho$ is large, the second term in (26) becomes dominant:

$$
f_{\text {eff }}(\rho) \rightarrow B^{\prime} \rho^{\beta}
$$

On the other hand, if $\beta<\alpha$, the second term becomes dominant and we obtain (27) again when $\rho \rightarrow 0$. In case of (12) without $G(H)$, when $1 / 2<\alpha<1$, there is the type I singularity where $\rho$ goes to infinity in a finite time. When $G(H)$ is given by (25), if $\beta>\alpha$, the second term in (26) becomes dominant and therefore if $\beta>1$, instead of type I singularity there occurs type III singularity. In case of (12) with $\alpha>1$, the type III singularity appears before $G(H)$ is included. Even if we include $G(H)$ with $\beta>\alpha>1$, we obtain the type III singularity again and the structure of the singularity is not changed qualitatively. For (22) without $G(H)$, when $0<\alpha<1 / 2$ or $\alpha<0$, there appears the type IV or type II singularity where $\rho$ tends to zero. Since the second term becomes dominant if $\beta<\alpha$, if $\beta<0$, the type IV singularity for $0<\alpha<1 / 2$ case becomes the type II singularity but the type II singularity for $\alpha<0$ is not qualitatively changed.

In accordance with the previous cases, one finds

- In case $\alpha>1$, for most values of $\beta$, there occurs type III singularity. In addition to the type III singularity, when $0<\beta<1 / 2$, there occurs type IV singularity and when $\beta<0$, there occurs type II singularity.

- $\alpha=1$ case, if $\beta>1$, the singularity becomes type III. $\beta=1$ case corresponds to (22). If $\beta<1$ and $A>0$, there occurs the Big Rip or type I singularity. In addition to the type I singularity, we have type IV singularity when $0<\beta<1 / 2$ and type II when $\beta<1$.

- In case $1 / 2<\alpha<1$, one sees singularity of type III for $\beta>1$, type I for $1 / 2 \leq \beta<1$ (even for $\beta=1 / 2$ ) or $\beta=1$ and $B^{\prime}>0(B>0)$ case. In addition to type I, type IV case occurs for $0<\beta<1 / 2$, and type II for $\beta<0$.
- In case $\alpha=1 / 2$, we have singularity of type III for $\beta>1$, type $\mathrm{I}$ for $1 / 2<\beta<1$ or $\beta=1$ and $B^{\prime}>0$ $(B>0)$, type IV for $0<\beta<1 / 2$, and type II for $\beta<0$. When $\beta=1 / 2$ or $\beta=0$, there does not appear any singularity.

- In case $0<\alpha<1 / 2$, we find type IV for $0<\beta<$ $1 / 2$, and type II for $\beta<0$. In addition to type IV singularity, there occurs singularity of type III for $\beta>1$, type $\mathrm{I}$ for $1 / 2 \leq \beta<1$ or $\beta=1$ and $B^{\prime}>0$ $(B>0)$ case.

- In case $\alpha<0$, there will always occur type II singularity. In addition to type II singularity, we have a singularity of type III for $\beta>1$, type I for $1 / 2 \leq \beta<1$ or $\beta=1$ and $B^{\prime}>0(B>0)$ case.

Thus, we demonstrated how the modification of EOS by Hubble dependent, inhomogeneous term changes the structure of singularity in late-time dark energy universe.

We now consider general case and assume $F(\rho)$ in (20) behaves as

$$
F(\rho) \sim F_{0} \rho^{\alpha}
$$

with constant $F_{0}$ and $\alpha$ in a proper limit (e.g. for large $\rho$ or small $\rho$ ). Then when $\alpha \neq 1$, Eq.(20) can be integrated as

$$
F_{0}\left(t-t_{c}\right) \sim \frac{\rho^{1-\alpha}}{1-\alpha}
$$

that is,

$$
\rho \sim\left((1-\alpha) F_{0}\left(t-t_{c}\right)\right)^{\frac{1}{1-\alpha}} .
$$

Here $t_{c}$ is a constant of the integration. When $\alpha=1$, the energy becomes

$$
\rho=\rho_{0} \mathrm{e}^{F_{0} t}
$$

with a constant of integration $\rho_{0}$. By using the first FRW equation (5), the scale factor may be found

$$
a=a_{0} \mathrm{e}^{ \pm \frac{2 \kappa}{(3-2 \alpha) \sqrt{3} F_{0}}\left((1-\alpha) F_{0}\left(t-t_{c}\right)\right)^{\frac{3-2 \alpha}{2(1-\alpha)}}},
$$

when $\alpha \neq 1$ and

$$
a=a_{0} \mathrm{e}^{\frac{2 \kappa}{F_{0}} \sqrt{\frac{\rho_{0}}{3}} \mathrm{e}^{\frac{F_{0}}{2}}}
$$

when $\alpha=1$.

In [4], there has been given an explicit example of the EOS where crossing of $w=-1$ phantom divide occurs:

$$
a(t)=a_{0}\left(\frac{t}{t_{s}-t}\right)^{n} .
$$

Here $n$ is a positive constant and $0<t<t_{s}$. The scale factor diverges in a finite time $\left(t \rightarrow t_{s}\right)$ as in the Big Rip. Therefore $t_{s}$ corresponds to the life time of the universe. When $t \ll t_{s}, a(t)$ evolves as $t^{n}$, which means that the 
effective EOS is given by $w=-1+2 /(3 n)>-1$. On the other hand, when $t \sim t_{s}$, it appears $w=-1-2 /(3 n)<$ -1 . The solution (34) has been obtained with

$$
f(\rho)= \pm \frac{2 \rho}{3 n}\left\{1-\frac{4 n}{t_{s}}\left(\frac{3}{\kappa^{2} \rho}\right)^{\frac{1}{2}}\right\}^{\frac{1}{2}}
$$

Therefore the EOS needs to be double-valued in order for the transition to occur between the region $w<-1$ and the region $w>-1$. Then in general, there could not be one-to-one correspondence between $p$ and $\rho$ in the above EOS. In such a case, instead of (18), we may suggest the implicit, inhomogeneous equation of the state

$$
F(p, \rho, H)=0
$$

The following example may be of interest:

$$
(p+\rho)^{2}-C_{0} \rho^{2}\left(1-\frac{H_{0}}{H}\right)=0 .
$$

Here $C_{0}$ and $H_{0}$ are positive constants. Combining (37) with the energy conservation law (19) and the first FRW equation (5), one can delete $p$ and $\rho$ as

$$
\dot{H}^{2}=\frac{9}{4} C_{0} H^{4}\left(1-\frac{H_{0}}{H}\right),
$$

which can be integrated as

$$
H=\frac{16}{9 C_{0}^{2} H_{0}\left(t-t_{-}\right)\left(t_{+}-t\right)} .
$$

Here

$$
t_{ \pm}=t_{0} \pm \frac{4}{3 C_{0} H_{0}}
$$

and $t_{0}$ is a constant of the integration. Hence

$$
\begin{aligned}
& p=-\rho\left\{1+\frac{3 C_{0}^{2}}{4 H_{0}}\left(t-t_{0}\right)\right\}, \\
& \rho=\frac{2^{8}}{3^{3} C_{0}^{4} H_{0}^{2} \kappa^{2}\left(t-t_{-}\right)^{2}\left(t_{+}-t\right)^{2}} .
\end{aligned}
$$

In (39), since $t_{-}<t_{0}<t_{+}$, as long as $t_{-}<t<t_{+}$, the Hubble rate $H$ is positive. The Hubble rate $H$ has a minimum $H=H_{0}$ when $t=t_{0}=\left(t_{-}+t_{+}\right) / 2$ and diverges when $t \rightarrow t_{ \pm}$. Then we may regard $t \rightarrow t_{-}$as a Big Bang singularity and $t \rightarrow t_{+}$as a Big Rip one. As clear from (41), the parameter $w=p / \rho$ is larger than -1 when $t_{-}<t<t_{0}$ and smaller than -1 when $t_{0}<t<t_{+}$. Therefore there occurs crossing of phantom divide $w=$ -1 when $t=t_{0}$ thanks to the effect of inhomogeneous term in EOS.

One more example may be of interest:

$$
(\rho+p)^{2}+\frac{16}{\kappa^{4} t_{0}^{2}}\left(h_{0}-H\right) \ln \left(\frac{h_{0}-H}{h_{1}}\right)=0 .
$$

Here $t_{0}, h_{0}, h_{1}$ are constants and $h_{0}>h_{1}>0$. A solution is given by

$$
\begin{aligned}
& H=h_{1}-h_{1} \mathrm{e}^{-t^{2} / t_{0}^{2}}, \quad \rho=\frac{3}{\kappa^{2}}\left(h_{1}-h_{1} \mathrm{e}^{-t^{2} / t_{0}^{2}}\right)^{2} \\
& p=-\frac{3}{\kappa^{2}}\left(h_{1}-h_{1} \mathrm{e}^{-t^{2} / t_{0}^{2}}\right)^{2}-\frac{4 h_{1} t}{\kappa^{2} t_{0}^{2}} \mathrm{e}^{-t^{2} / t_{0}^{2}}
\end{aligned}
$$

Hence,

$$
\dot{H}=\frac{2 h_{1} t}{t_{0}^{2}} \mathrm{e}^{-t^{2} / t_{0}^{2}} .
$$

Using the energy conservation law (19) and the first FRW equation (5), the second FRW equation may be found:

$$
-\frac{2}{\kappa^{2}} \dot{H}=\rho+p
$$

As in (44), $\dot{H}$ is negative when $t<0$ and positive when $t>0$. Eq. 45) tells that the effective parameter $w=p / \rho$ of the equation of the state is $w>-1$ when $t<0$ and $w<-1$ when $t>0$. As we find the Hubble rate $H$ goes to a constant $h_{0}, H \rightarrow h_{0}$, in the limit of $t \rightarrow \pm \infty$, the universe asymptotically approaches to deSitter phase. Therefore there does not appear Big Rip nor Big Bang singularity.

Hence, we presented several examples of inhomogeneous EOS for ideal fluid and demonstrated how the final state of the universe filled with such fluid changes if compare with homogeneous case. The ideal fluid with implicit EOS may be used to construct the cosmologies which cross the phantom divide.

The interesting remark is in order (see also Appendix). In principle, the more general EOS may contain the derivatives of $H$, like $\dot{H}, \ddot{H}, \ldots$ Then more general EOS than (36) has the following form:

$$
F(p, \rho, H, \dot{H}, \ddot{H}, \cdots)=0 .
$$

Trivial example is that

$$
p=w \rho-\frac{2}{\kappa^{2}} \dot{H}-\frac{3(1+w)}{\kappa^{2}} H^{2} .
$$

By using the first (5) or second (45) FRW equations, we find

$$
\rho=\frac{3}{\kappa^{2}} H^{2}, \quad p=-\frac{2}{\kappa^{2}} \dot{H}-\frac{3}{\kappa^{2}} H^{2} .
$$

Therefore Eq.(47) becomes an identity, which means that any cosmology can be a solution if EOS (47) is assumed.

Another, non-trivial example is

$$
p=w \rho-G_{0}-\frac{2}{\kappa^{2}} \dot{H}+G_{1} \dot{H}^{2} .
$$

Here it is supposed $G_{0}(1+w)>0$. If $G_{1}(1+w)>0$, there appears a solution which describes an oscillating universe,

$$
H=h_{0} \cos \omega t, \quad a=a_{0} \mathrm{e}^{\frac{h_{0}}{\omega} \sin \omega t} .
$$


Here

$$
h_{0} \equiv \kappa \sqrt{\frac{G_{0}}{3(1+w)}}, \quad \omega=\sqrt{\frac{3(1+w)}{G_{1} \kappa^{2}}} .
$$

In case $G_{1}(1+w)<0$, another cosmological solution appears

$$
H=h_{0} \cosh \tilde{\omega} t, \quad a=a_{0} \mathrm{e}^{\frac{h_{0}}{\omega} \sinh \tilde{\omega} t} .
$$

Here $h_{0}$ is defined by (51) again and $\tilde{\omega}$ is defined by

$$
\tilde{\omega}=\sqrt{-\frac{3(1+w)}{G_{1} \kappa^{2}}} .
$$

One can go further and present many more examples of inhomogeneous EOS cosmology.

\section{FRW COSMOLOGY WITH INHOMOGENEOUS INTERACTING FLUIDS}

In the present section, we study FRW universe filled with two interacting fluids. Note that there is some interest to study the cosmology with homogeneous interacting fluids [4, 15]. The inhomogeneous terms for such cosmology may be again motivated by (bulk) viscosity account [16].

Let us consider a system with two fluids, which satisfy the following EOS:

$$
p_{1,2}=-\rho_{1,2}-f_{1,2}\left(\rho_{1,2}\right)-G_{1,2}(H)
$$

For simplicity, the only case is considered that

$$
p_{ \pm}=w_{ \pm} \rho_{ \pm}-G_{ \pm}(H)
$$

In the above equation and in the following, the indexes \pm instead of 1,2 , as $p_{1,2}=p_{ \pm}$are used. In a spatially flat FRW universe with a scale factor $a$, the cosmological equations are given by

$$
\begin{aligned}
& \dot{\rho}_{ \pm}+3 H\left(\rho_{ \pm}+p_{ \pm}\right)=\mp Q \\
& \dot{H}=-\frac{\kappa^{2}}{2}\left(\rho_{+}+p_{+}+\rho_{-}+p_{-}\right) \\
& H^{2}=\frac{\kappa^{2}}{3}\left(\rho_{+}+\rho_{-}\right)
\end{aligned}
$$

Not all of the above equations are independent, for example, Eqs.(56) and (58) lead to (57). From Eqs.(58), (57), and the equation for $\rho_{+}$and $p_{+}$of (56), one obtains the equation for $\rho_{-}$and $p_{-}$of (56). In [4], the following case has been considered:

$$
G_{ \pm}(H)=0, \quad Q=\delta H^{2}, \quad w_{+}=0, \quad w_{-}=-2
$$

where $\delta$ is a constant. Then combining Eq. (58) with Eqs. (56), the explicit solution follows

$$
\begin{aligned}
H & =\frac{2}{3}\left(\frac{1}{t}+\frac{1}{t_{s}-t}\right) \\
\rho_{+} & =\frac{4}{3 \kappa^{2}}\left(\frac{1}{t}+\frac{1}{t_{s}-t}\right) \frac{1}{t}, \\
\rho_{-} & =\frac{4}{3 \kappa^{2}}\left(\frac{1}{t}+\frac{1}{t_{s}-t}\right) \frac{1}{t_{s}-t},
\end{aligned}
$$

where

$$
t_{s} \equiv \frac{9}{\delta \kappa^{2}}
$$

In (60), it is assumed $0<t<t_{s}$. The Hubble rate $H$ diverges in a finite time $\left(t \rightarrow t_{s}\right)$ as in the Big Rip singularity. Therefore $t_{s}$ corresponds to the life time of the universe. When $t \ll t_{s}, H$ behaves as $2 / 3 t$, which means that the effective EOS is given by $w_{\text {eff }} \sim 0>-1$. On the other hand, when $t \sim t_{s}$, it appears $w_{\text {eff }}=-2<$ -1 . Therefore the crossing of phantom divide $w_{\text {eff }}=-1$ occurs.

From (55- 58), we obtain

$$
\begin{aligned}
\rho_{ \pm}= & \frac{3}{2 \kappa^{2}} H^{2} \\
& \pm \frac{1}{w_{+}-w_{-}}\left\{G_{+}(H)+G_{-}(H)\right. \\
& \left.-\frac{3}{\kappa^{2}}\left(1+\frac{w_{+}+w_{-}}{2}\right) H^{2}-\frac{2}{\kappa^{2}} \dot{H}\right\} \\
Q= & -\frac{1}{w_{+}-w_{-}}\left\{\left(G_{+}^{\prime}(H)+G_{-}^{\prime}(H)\right) \dot{H}\right. \\
& \left.-\frac{6}{\kappa^{2}}\left(1+\frac{w_{+}+w_{-}}{2}\right) \dot{H}_{-} \frac{2}{\kappa^{2}} \ddot{H}\right\} \\
& +3 H\left(1+\frac{w_{+}+w_{-}}{2}\right) \\
& \times \frac{1}{w_{+}-w_{-}}\left\{G_{+}(H)+G_{-}(H)\right. \\
& \left.-\frac{3}{\kappa^{2}}\left(1+\frac{w_{+}+w_{-}}{2}\right) H^{2}-\frac{2}{\kappa^{2}} \dot{H}\right\} \\
& -\frac{9\left(w_{+}-w_{-}\right)}{4 \kappa^{2}} H^{3} \\
& -\frac{3}{2} H\left(G_{+}(H)-G_{-}(H)\right) .
\end{aligned}
$$

First, the case is considered that the Hubble rate $H$ satisfies the following equation:

$$
\dot{H}=S(H)
$$

where $S(H)$ is a proper function of $H$. Hence, $Q$ can be 
presented as a function of $H$ as

$$
\begin{aligned}
Q= & Q(H) \\
= & -\frac{1}{w_{+}-w_{-}}\left\{\left(G_{+}^{\prime}(H)+G_{-}^{\prime}(H)\right) S(H)\right. \\
& \left.+3\left(1+\frac{w_{+}+w_{-}}{2}\right) H\left(G_{+}(H)+G_{-}(H)\right)\right\} \\
& +\frac{12}{\kappa^{2}\left(w_{+}-w_{-}\right)}\left(1+\frac{w_{+}+w_{-}}{2}\right) H S(H) \\
& -\frac{9}{\kappa^{2}\left(w_{+}-w_{-}\right)} \\
& \times\left\{\left(1+\frac{w_{1}+w_{2}}{2}\right)^{2}+\frac{\left(w_{+}-w_{-}\right)^{2}}{4}\right\} H^{3} \\
& +\frac{2}{\kappa^{2}\left(w_{+}-w_{-}\right)} S^{\prime}(H) S(H) \\
& -\frac{3}{2} H\left(G_{+}(H)-G_{-}(H)\right) .
\end{aligned}
$$

If $Q$ is given by (67) for proper $G_{p}(H)$ and $S(H)$, the solution of Eqs. (55 - 58) can be obtained by solving Eq. (66) with respect to $H$. Then from (64), one finds the behavior of $\rho_{ \pm}$. As an example, if we consider $S(H)$ given by

$$
S(H)=-\frac{1}{h_{1}}\left(H-h_{0}\right),
$$

the solution of (67) is given by

$$
H=h_{0}+\frac{h_{1}}{t-t_{0}},
$$

Here $t_{0}$ is a constant of the integration. In the solution (69), as $H$ behaves as $H \sim \frac{h_{1}}{t-t_{0}}$ when $t-t_{0} \sim 0$, the effective $w_{\text {eff }}$ is given by $w=-1+\frac{2}{3 h_{1}}$. On the other hand, as $H$ becomes a constant $h_{0}$ when $t$ is large, we obtain the effective $w_{\text {eff }}=1$.

Next the simpler case is considered:

$$
w_{ \pm}=-1 \pm w, \quad G_{ \pm}(H)= \pm G(H) .
$$

Then (64) and 65) have the following forms:

$$
\begin{aligned}
\rho_{ \pm} & =\frac{3}{2 \kappa^{2}} H^{2} \mp \frac{1}{\kappa^{2} w} \dot{H}, \\
Q & =\frac{1}{\kappa^{2} w} \ddot{H}-\frac{9 w}{2 \kappa^{2}} H^{3}-3 H G(H) .
\end{aligned}
$$

Thus, for example, for an arbitrary $G(H)$, if $Q$ is given by a function of $H$ as

$$
Q=\frac{\omega^{2}}{\kappa^{2} w}\left(H-h_{0}\right)-\frac{9 w}{2 \kappa^{2}} H^{3}-3 H G(H),
$$

that is,

$$
\ddot{H}=\omega^{2}\left(h_{0}-H\right),
$$

the solution of Eqs.(55)- 58 is given by

$$
\begin{aligned}
H= & h_{0}+h_{1} \sin (\omega t+\alpha), \\
\rho_{ \pm}= & \frac{3}{2 \kappa^{2}}\left(h_{0}+h_{1} \sin (\omega t+\alpha)\right)^{2} \\
& \mp \frac{h_{1} \omega}{\kappa^{2} w} \cos (\omega t+\alpha) .
\end{aligned}
$$

Here $h_{1}$ and $\alpha$ are constants of the integration. This demonstrates how the inhomogeneous term modifies latetime cosmology.

Choosing $G_{ \pm}(H)$ and $Q$, one may realize a rather general cosmology. As was shown, if we introduce two fluids, even without assuming the non-linear EOS as in (36), the model crossing $w=-1$ effectively can be realized. In fact, from (75) one has

$$
\dot{H}=h_{1} \omega \cos (\omega t+\alpha)
$$

which changes its sign depending on time. When $\dot{H}>0$, effectively $w<-1$, and when $\dot{H}<0, w>-1$. Note that, as a special case in (73), we may choose,

$$
G(H)=\frac{\omega^{2}}{3 \kappa^{2} w}\left(1-\frac{h_{0}}{H}\right)-\frac{3 w}{2 \kappa^{2}} H^{2},
$$

which gives $Q=0$. As $Q=0$, from (56), there is no direct interaction between two fluids. As is clear from (75), however, there is an oscillation in the energy densities, which may indicate that there is a transfer of the energy between the fluids. Hence, the $G(H)$ term might generate indirect transfer between two fluids.

\section{CROSSING THE PHANTOM BARRIER WITH INHOMOGENEOUS EOS AND THERMODYNAMICAL CONSIDERATIONS}

Let us start from the EOS (9). Assuming that $w$ crosses -1 , which corresponds to $f(\rho)=0$, in order that the integrations in (10) and (11) are finite, $f(\rho)$ should behave as

$$
f(\rho) \sim f_{0}\left(\rho-\rho_{0}\right)^{s}, \quad 0<s<1 .
$$

Here $f\left(\rho_{0}\right)=0$. Since $0<s<1, f(\rho)$ could be multivalued at $\rho=\rho_{0}$, in general. Near $\rho=\rho_{0}$, Eq.(11) gives,

$$
t-t_{0} \sim \frac{\left(\rho-\rho_{0}\right)^{1-s}}{\kappa \sqrt{3} \rho_{0} f_{0}(1-s)} .
$$

Here $t=t_{0}$ when $\rho=\rho_{0}$. Since

$$
\dot{H}=\frac{\kappa^{2}}{2} f(\rho),
$$

from the second FRW Eq.(45), one finds

$$
\begin{aligned}
& \dot{H} \sim \frac{\kappa}{2^{2}} f_{0}\left(\frac{t-t_{0}}{t_{1}}\right)^{s /(1-s)}, \\
& t_{1} \equiv \frac{1}{\kappa \sqrt{3} \rho_{0} f_{0}(1-s)} .
\end{aligned}
$$


Hence, when $s /(1-s)$ is positive odd integer, the sign of $\dot{H}$ changes at $t=t_{0}$, which shows the crossing $w=-1$.

In recent paper 13], based on consideration of mixture of two fluids: effective quintessence and effective phantom, the following, quite interesting EOS has been suggested:

$$
A \rho^{m}+B p^{m}=\left(C \rho^{m}+D p^{m}\right)^{\alpha} .
$$

Here $A, B, C, D$, and $\alpha$ are constants and $m$ is an integer. This EOS can be regarded as a special case of (36). By writing $p$ as

$$
p=Q(\rho) \rho
$$

one obtains

$$
\rho^{m(\alpha-1)}=F\left(Q^{m}\right) \equiv\left(A+B Q^{m}\right)\left(C+D Q^{m}\right)^{-\alpha} .
$$

Since

$$
\begin{aligned}
F^{\prime}\left(Q^{m}\right)= & \left(C+D Q^{m}\right)^{-\alpha-1} \\
& \times\left(B C-\alpha A D+(1-\alpha) B D Q^{m}\right)
\end{aligned}
$$

it follows $F^{\prime}\left(Q^{m}\right)=0$ when

$$
Q^{m}=-\frac{\frac{C}{D}-\alpha \frac{A}{B}}{1-\alpha} .
$$

By properly choosing the parameters, we assume

$$
\frac{\frac{C}{D}-\alpha \frac{A}{B}}{1-\alpha}=1
$$

When $Q \sim-1$,

$$
F\left(Q^{m}\right) \sim q_{0}+q_{2}(Q+1)^{2} .
$$

Here

$$
\begin{aligned}
q_{0} & =F(1)=(A-B)(C+D)^{-\alpha}, \\
q_{2} & =\left.\frac{1}{2} \frac{d^{2} F}{d Q^{2}}\right|_{Q=-1} \\
& =-\alpha(\alpha-1)(C-D)^{-\alpha-2} D^{2}(A-B) m^{2} .
\end{aligned}
$$

In (89), it is supposed $m$ is an odd integer. Solving (84) with (88) with respect to $Q$, one arrives at

$$
Q=-1 \pm\left\{\frac{m(\alpha-1) \rho_{0}^{m \alpha-m-1}\left(\rho-\rho_{0}\right)}{q_{2}}\right\}^{1 / 2}
$$

Here $\rho_{0}$ is defined by

$$
q_{0}=\rho_{0}^{m(\alpha-1)} .
$$

Using (83), the function $\mathrm{Q}$ is

$$
p \sim-\rho \pm \rho_{0}\left\{\frac{m(\alpha-1) \rho_{0}^{m \alpha-m-1}\left(\rho-\rho_{0}\right)}{q_{2}}\right\}^{1 / 2} .
$$

Comparing (92) with (78), we find that the EOS (82) surely corresponds to $s=1 / 2$ case in (78).

For the EOS (82), there are interesting, exactly solvable cases. We now consider such a case and see that there are really the cases of EOS crossing barrier $w=-1$. The energy conservation law (2) may be rewritten as follows:

$$
p=-\rho-V \frac{d \rho}{d V}, \quad V \equiv V_{0} a^{3} .
$$

Here $V_{0}$ is a constant with the dimension of the volume. Use of Eq. (83) gives

$$
0=V \frac{d \rho}{d V}+(1+Q(\rho)) \rho .
$$

Using (84), we further rewrite (94) as an equation with respect to $Q$ :

$$
\begin{aligned}
0= & -\frac{\left(B C-\alpha A D+B D(1-\alpha) Q^{m}\right) Q^{m-1}}{(1-\alpha)\left(C+D Q^{m}\right)\left(A+B Q^{m}\right)} V \frac{d Q}{d V} \\
& +1+Q
\end{aligned}
$$

Assuming Eq.(87), the above Eq.(95) takes a simple form:

$$
0=-\frac{B D\left(1+Q^{m}\right) Q^{m-1}}{\left(C+D Q^{m}\right)\left(A+B Q^{m}\right)} V \frac{d Q}{d V}+1+Q .
$$

Especially in the simplest case $m=1$, one can easily solve (96)

$$
Q=-\frac{C-A\left(\frac{V}{V_{1}}\right)^{\beta}}{D-B\left(\frac{V}{V_{1}}\right)^{\beta}} .
$$

Here $V_{1}$ is a constant of the integration and

$$
\beta \equiv \frac{B D}{A D-B C}=\frac{1}{(1-\alpha)\left(\frac{A}{B}-1\right)} .
$$

In the above equation, Eq.87) is used. Hence, when $\left(V / V_{1}\right)^{\beta} \rightarrow 0$, it follows $w=p / \rho=Q \rightarrow-C / D$. On the other hand, when $\left(V / V_{1}\right)^{\beta} \rightarrow \infty$, one arrives at $w=$ $Q \rightarrow-A / B$. Hence, the value of $w$ changes depending on the size of the universe. Especially when

$$
\frac{V}{V_{1}}=\left(\frac{C-D}{A-B}\right)^{1 / \beta}
$$

there occurs the crossing of phantom divide $w=Q=-1$ (compare with [13]).

As the inhomogeneous generalization of the EOS (82), we may consider

$$
A\left(\frac{3}{\kappa^{2}} H^{2}\right)^{m}+B p^{m}=\left(C \rho^{m}+D p^{m}\right)^{\alpha},
$$

or

$$
A \rho^{m}+B p^{m}=\left(C\left(\frac{3}{\kappa^{2}} H^{2}\right)^{m}+D p^{m}\right)^{\alpha}
$$


or, more general EOS

$$
\begin{array}{r}
\left(A-A^{\prime}\right) \rho^{m}+A^{\prime}\left(\frac{3}{\kappa^{2}} H^{2}\right)^{m}+B p^{m} \\
=\left(\left(C-C^{\prime}\right) \rho^{m}+C^{\prime}\left(\frac{3}{\kappa^{2}} H^{2}\right)^{m}+D p^{m}\right)^{\alpha} .
\end{array}
$$

By using the first FRW equation (5), it folows that the EOS (100), (101), and (102) are equivalent to (82). Especially if $m=1$ and (87) could be satisfied, one obtains the solution (97).

Hence, using the first and second FRW Eqs.(5) and (45), the EOS (82) with $m=1$ can be rewritten as

$$
\begin{aligned}
& \frac{d^{2}}{d t^{2}}\left(a^{\frac{3}{2}\left(1-\frac{A}{B}\right)}\right) \\
& =\frac{3 \kappa^{2}(A-B)}{4 B^{2}}\left(\frac{3 \kappa^{2}(C-D)}{4 D^{2}}\right)^{-\alpha} \\
& \quad \times a^{\frac{3}{2}\left\{1-\frac{A}{B}-\alpha\left(1-\frac{C}{D}\right)\right\}}\left\{\frac{d^{2}}{d t^{2}}\left(a^{\frac{3}{2}\left(1-\frac{C}{D}\right)}\right)\right\} .
\end{aligned}
$$

When (87) is satisfied, this second order differential Eq. looks as

$$
\begin{aligned}
\frac{d^{2} X}{d t^{2}} & =\left(\frac{4 B}{3 \kappa^{2}(A-B)}\right)^{\alpha-1} \alpha^{\alpha}\left(\frac{d^{2} X^{\frac{1}{\alpha}}}{d t^{2}}\right)^{\alpha}, \\
X & \equiv a^{\frac{3}{2}\left(1-\frac{A}{B}\right)},
\end{aligned}
$$

which also admits, besides the solution crossing $w=-1$ (97), a flat universe solution

$$
a=a_{0}, \quad\left(a_{0}: \text { constant }\right),
$$

and deSitter universe solution

$$
a=a_{0} \mathrm{e}^{\frac{2}{\kappa} \sqrt{\frac{B}{A-B}} \alpha^{\frac{\alpha}{2(1-\alpha)}} t} .
$$

As next generalization of (82), one may consider the following EOS:

$$
\begin{aligned}
& A \rho+B p-\frac{A-B}{\kappa^{2}} H^{2} \\
& =\left(C \rho+D p-\frac{C-D}{\kappa^{2}} H^{2}\right)^{\alpha(H)} .
\end{aligned}
$$

Here $\alpha$ is assumed to be a function of $H$. Then by using the first and second FRW Eqs.(5) and (45), the EOS 107) can be rewritten as

$$
-\frac{2 B}{\kappa^{2}} \dot{H}=\left(-\frac{2 D}{\kappa^{2}} \dot{H}\right)^{\alpha(H)},
$$

which gives

$$
-\frac{\kappa^{2}}{2 D} t=\int^{H} d H \mathrm{e}^{-\frac{\ln \frac{B}{D}}{\alpha(t)-1}}
$$

As an example, for the solution (75)

$$
\omega t=\frac{1}{h_{1}} \int^{H} \frac{d H}{\sqrt{1-\left(\frac{H-h_{0}}{h_{1}}\right)^{2}}} .
$$

Comparing (109) with (110), in case that

$$
h_{1} \omega=-\frac{\kappa^{2}}{2 D}, \quad \alpha(H)=1+\frac{2 \ln \frac{B}{D}}{\ln \left(1-\left(\frac{H-h_{0}}{h_{1}}\right)^{2}\right)},
$$

the solution (75) follows from the EOS (107).

As another generalization of (82), we may consider the following EOS:

$$
A \rho^{m}+B p^{m}=G(H)\left(C \rho^{m}+D p^{m}\right)^{\alpha} .
$$

Here $G(H)$ is a function of the Hubble rate. For simplicity, the following case is considered

$$
m=1, \quad G(H)=\left(\frac{3}{\kappa^{2}} H^{2}\right)^{\gamma} .
$$

Then, writing $p$ as (83) and using $Q$, the energy looks like

$$
\rho=(A+B Q)^{\frac{1}{\gamma+\alpha-1}}(C+D Q)^{-\frac{\alpha}{\gamma+\alpha-1}},
$$

which corresponds to (84). Assuming Eq.(87), by using (83), instead of (96), one gets

$$
0=-\frac{(1-\alpha) B D}{(1-\alpha-\gamma)(C+D Q)(A+B Q)} V \frac{d Q}{d V}+1,
$$

which can be solved as

$$
Q=-\frac{C-A\left(\frac{V}{V_{1}}\right)^{\tilde{\beta}}}{D-B\left(\frac{V}{V_{1}}\right)^{\tilde{\beta}}} .
$$

Here $V_{1}$ is again a constant of the integration and

$$
\tilde{\beta} \equiv \frac{(1-\alpha) B D}{(1-\alpha-\gamma) A D-B C} .
$$

Then as in (97), when $\left(V / V_{1}\right)^{\beta} \rightarrow 0$, we have $w=$ $p / \rho=Q \rightarrow-C / D$ and when $\left(V / V_{1}\right)^{\beta} \rightarrow \infty$, we have $w=Q \rightarrow-A / B$. The power of $V$, however, is changed in Eq. (116) if compare with Eq. (97). Thus, we presented number of FRW cosmologies (including oscillating universes) filled by cosmic fluid with inhomogeneous EOS where phantom divide is crossing. Definitely, one can suggest more examples or try to fit the astrophysical data with more precise model of above sort.

In [17], the thermodynamical models of the dark energy have been constructed. Especially it has been shown that, for the fluid with constant $w$, the free energy $F(T, V)$ is generally given by

$$
F(T, V)=T \hat{F}\left(\left(T / T_{0}\right)^{1 / w}\left(V / V_{0}\right)\right) .
$$


Here $T$ is the temperature and $V$ is the volume of the universe. For the dimensional reasons, the positive parameters $T_{0}$ and $V_{0}$ are introduced.

The interesting question is what happens with the entropy when the value of $w$ crosses -1 . As a model, the case that $w=Q$ depends on $V$ as in (97) may be considered:

$$
w=w(V)=\frac{w_{0}+w_{1}\left(\frac{V}{V_{0}}\right)^{\beta}}{1+\left(\frac{V}{V_{0}}\right)^{\beta}} .
$$

When $\beta>0, w \rightarrow w_{0}$ for small universe and $w \rightarrow w_{1}$ for large universe.

The specific dependence of free energy may be taken as below

$$
F=\frac{f_{0} T}{T_{0}}\left\{\left(\frac{T}{T_{0}}\right)^{\frac{1}{w(V)}} \frac{V}{V_{0}}\right\}^{\gamma} .
$$

Here $\gamma$ is a constant. When $\gamma=1$ and $w$ is a constant, the free energy is proportional to the volume. For usual matter, due to self-interaction and related effects, $\gamma$ is not always unity. Then, the pressure $p$, the energy density $\rho$, and the entropy $\mathcal{S}$ are given by

$$
\begin{aligned}
p= & -\frac{\partial F}{\partial V} \\
= & -\frac{f_{0} \gamma}{V_{0}}\left(\frac{T}{T_{0}}\right)^{1+\frac{\gamma}{w(V)}}\left(\frac{V}{V_{0}}\right)^{\gamma-1} \\
& \times\left\{1+\gamma \ln \left(\frac{T}{T_{0}}\right) \frac{\left(w_{1}-w_{0}\right) \beta\left(\frac{V}{V_{0}}\right)^{\beta}}{\left(w_{0}+w_{1}\left(\frac{V}{V_{0}}\right)^{\beta}\right)^{2}}\right\}, \\
\rho= & \frac{1}{V}\left(F-T \frac{\partial F}{\partial T}\right) \\
= & -\frac{f_{0} \gamma}{w V_{0}}\left(\frac{T}{T_{0}}\right)^{1+\frac{\gamma}{w(V)}}\left(\frac{V}{V_{0}}\right)^{\gamma-1}, \\
\mathcal{S} & -\frac{\partial F}{\partial T} \\
= & -\frac{f_{0}}{T_{0}}\left(1+\frac{\gamma}{w}\right)\left(\frac{T}{T_{0}}\right)^{\frac{\gamma}{w(V)}}\left(\frac{V}{V_{0}}\right)^{\gamma} .
\end{aligned}
$$

In the pressure $p$, the second term in large \{\} comes from $V$ dependence of $w$ in (119), which vanishes for large or small universe $(V \rightarrow \infty$ or $V \rightarrow 0)$. Hence, for small or large universe $p / \rho \rightarrow w(V) \rightarrow w_{0,1}$. As seen from the expression for $\mathcal{S}$, the sign of the entropy changes at

$$
w=-\gamma \text {. }
$$

If $\gamma=1$, the sign of the entropy $\mathcal{S}$ changes when crossing $w=-1$ (the entropy becomes negative when $w$ is less than -1 as it was observed in [17]), but in the case that

$$
\gamma<\left|w_{0}\right|,\left|w_{1}\right|,
$$

the entropy does not change its sign.

We should note that the expressions (121) are not well-defined, unless $\gamma=0$, when $w=0$, which corresponds to dust. One may assume $0<\gamma<w_{0} \ll 1$ and $w_{1} \lesssim-1$. Then as clear from (119), $w$ changes from $w_{0} \sim 0$ for small universe to $w_{1} \lesssim-1$ for large universe and crosses -1 . Since we always have $\left|\gamma / w_{0}\right|<1$ and therefore $1+\gamma / w>0$, the entropy $\mathcal{S}$ (121) is always positive and does not change its sign as long as $f_{0}<0$. This explicitly demonstrates very beatiful phenomenon: there exist thermodynamical models for dark energy with crossing of phantom divide. Despite the preliminary expectations, the entropy of such dark energy universe even in its phantom phase may be positive!

\section{DISCUSSION}

In summary, the effect of modification of general EOS of dark energy ideal fluid by the insertion of inhomogeneous, Hubble parameter dependent term in the late-time universe is considered. Several explicit examples of such term which is motivated by time-dependent bulk viscosity or deviations from general relativity are considered. The corresponding late-time FRW cosmology (mainly, in its phantom epoch) is described. It is demonstrated how the structure of future singularity is changed thanks to generalization of dark energy EOS. The number of FRW cosmologies admitting the crossing of phantom barrier are presented. The inhomogeneous term in EOS helps to realize such a transition in a more natural way.

It is interesting that in the case when universe is filled with two interacting fluids (for instance, dark energy and dark matter) the Hubble parameter dependent term may effectively absorb the coupling between the fluids. Again, in case of two dark fluids the phantom epoch with possibility of crossing of $w=-1$ barrier occurs is constructed. It is also very interesting that there exists thermodynamical dark energy model where despite the preliminary expectations 17 the entropy in phantom epoch may be positive. This is caused by crossing of phantom barrier.

As it was demonstrated making the dark energy EOS more general, this extra freedom in inhomogeneous term brings a number of new possibilities to construct the latetime universe. One can go even further, assuming that inhomogeneous terms in EOS are not restricted by energy conservation law (as it is often the case in braneworld approach). Nevertheless, only more precise astrophysical data will help to understand which of number of EOS of the universe under consideration (in other words, dark energy models) is realistic.

\section{ACKNOWLEDGEMENTS}

We thank S. Tsujikawa for participation at the early stage of this work. The research by SDO has been partially supported by RFBR grant 03-01-00105 and LRSS 
grant 1252.2003 .2 .

\section{APPENDIX A: INHOMOGENEOUS TERMS FROM MODIFIED GRAVITY}

Let us consider the possibility to obtain the inhomogeneous EOS from the modified gravity. As an illustrative example, the following action is considered:

$$
S=\int d^{4} x \sqrt{-g}\left(\frac{1}{2 \kappa^{2}}+\mathcal{L}_{\text {matter }}+f(R)\right) .
$$

Here $f(R)$ can be an arbitrary function of the scalar curvature $R$ and $\mathcal{L}_{\text {matter }}$ is the Lagrangian for the matter. In the FRW universe, the gravitational equations are:

$$
\begin{aligned}
0= & -\frac{3}{\kappa^{2}} H^{2}+\rho-f\left(R=6 \dot{H}+12 H^{2}\right) \\
& +6\left(\dot{H}+H^{2}-H \frac{d}{d t}\right) \\
& \times f^{\prime}\left(R=6 \dot{H}+12 H^{2}\right), \\
0= & \frac{1}{\kappa^{2}}\left(2 \dot{H}+3 H^{2}\right)+p+f\left(R=6 \dot{H}+12 H^{2}\right) \\
& +2\left(-\dot{H}-3 H^{2}+\frac{d^{2}}{d t^{2}}+2 H \frac{d}{d t}\right) \\
& \times f^{\prime}\left(R=6 \dot{H}+12 H^{2}\right) .
\end{aligned}
$$

Here $\rho$ and $p$ are the energy density and the pressure coming from $\mathcal{L}_{\text {matter. }}$ They may satisfy the equation of state like $p=w \rho$. One may now define the effective energy density $\tilde{\rho}$ and $\tilde{p}$ by

$$
\begin{aligned}
\tilde{\rho} \equiv & \rho-f\left(R=6 \dot{H}+12 H^{2}\right) \\
& +6\left(\dot{H}+H^{2}-H \frac{d}{d t}\right) \\
& \times f^{\prime}\left(R=6 \dot{H}+12 H^{2}\right), \\
\tilde{p}= & p+f\left(R=6 \dot{H}+12 H^{2}\right) \\
& +2\left(-\dot{H}-3 H^{2}+\frac{d^{2}}{d t^{2}}+2 H \frac{d}{d t}\right) \\
& \times f^{\prime}\left(R=6 \dot{H}+12 H^{2}\right) .
\end{aligned}
$$

Thus, it follows

$$
\begin{aligned}
\tilde{p}= & w \tilde{\rho}+(1+w) f\left(R=6 \dot{H}+12 H^{2}\right) \\
& +2\left((-1-3 w) \dot{H}-3(1+w) H^{2}+\frac{d^{2}}{d t^{2}}\right. \\
& \left.+(2+3 w) H \frac{d}{d t}\right) f^{\prime}\left(R=6 \dot{H}+12 H^{2}\right)
\end{aligned}
$$

In the situation where the derivative of $H$ can be neglected as $\dot{H} \ll H^{2}$ or $\ddot{H} \ll H^{3}$, we find

$$
\begin{aligned}
\tilde{p} \sim & w \tilde{\rho}+G(H) \\
G(H) \equiv & (1+w) f\left(R=12 H^{2}\right) \\
& -3(1+w) H^{2} f^{\prime}\left(R=12 H^{2}\right) .
\end{aligned}
$$

Typically $H$ has a form like $H \sim h_{0} /\left(t-t_{1}\right)$ or $H \sim$ $h_{0} /\left(t_{2}-t\right)$, with $h_{0}=2 / 3(w+1)$, corresponding to (6). Hence, the condition $\dot{H} \ll H^{2}$ or $\ddot{H} \ll H^{3}$ requires $h_{0} \gg$ 1 , which shows $w \sim-1$ as in the modern universe. This supports our observation that inhomogeneous terms may be the effective ones which are predicted due to currently modified gravity theory.

The modification of the EOS by $G(H)$ terms might come also from the braneworld scenario. Indeed, the single brane model is described by the following simple action

$$
\begin{aligned}
S= & \frac{M_{\mathrm{Pl}}^{2}}{r_{c}} \int d^{4} x d y \sqrt{-g^{(5)}} R^{(5)} \\
& +\int d^{4} x \sqrt{-g}\left(M_{\mathrm{Pl}}^{2} R+\mathcal{L}_{\text {matter }}\right) .
\end{aligned}
$$

Here $M_{\mathrm{Pl}}^{2}=1 / 8 \pi G, y$ is the coordinate of the extra dimension, and $\mathcal{L}_{\text {matter }}$ is the Lagrangian density of the matters on the brane. The five-dimensional quantities are denoted by suffix "(5)". In ref. 18] it has been shown that the FRW equation for $4 \mathrm{~d}$ brane universe could be given by

$$
\frac{3}{\kappa^{2}}\left(H^{2} \pm \frac{H}{r_{c}}\right)=\rho
$$

Here $\rho$ is the matter energy density coming from $\mathcal{L}_{\text {matter }}$. More general case is considered in ref. [19] where the FRW equation is modified as

$$
\frac{3}{\kappa^{2}}\left(H^{2}-\frac{H^{\alpha}}{r_{c}^{2-\alpha}}\right)=\rho .
$$

Here $\alpha$ is a constant. One may assume that the matter energy density $\rho$ satisfies the energy conservation as in (2). Then from (A9), we find

$$
-\frac{2}{\kappa^{2}}\left(1-\frac{\alpha H^{\alpha-2}}{2 r_{c}^{2-\alpha}}\right) \dot{H}=\rho+p .
$$

By comparing (A10) with the first FRW equation (5) and A11 with the second FRW equation (45), one may define the effective energy density $\tilde{\rho}$ and pressure $\tilde{p}$ as

$$
\tilde{\rho} \equiv \rho+\frac{3 H^{\alpha}}{\kappa^{2} r_{c}^{2-\alpha}}, \quad \tilde{p} \equiv-\frac{3 H^{\alpha}}{\kappa^{2} r_{c}^{2-\alpha}}-\frac{\alpha H^{\alpha-2} \dot{H}}{\kappa^{2} r_{c}^{2-\alpha}}
$$

They satisfy the first (5) and second (45) FRW equations:

$$
\frac{3}{\kappa^{2}} H^{2}=\tilde{\rho}, \quad-\frac{2}{\kappa^{2}} \dot{H}=\tilde{\rho}+\tilde{p} .
$$


If it is also assumed the matter energy density $\rho$ and the matter pressure $p$ satisfy the EOS like $p=w \rho$, the effective EOS for $\tilde{\rho}$ and $\tilde{p}$ is given by

$$
\tilde{p}=w \tilde{\rho}-(1+w) \frac{3 H^{\alpha}}{\kappa^{2} r_{c}^{2-\alpha}}-\frac{\alpha H^{\alpha-2} \dot{H}}{\kappa^{2} r_{c}^{2-\alpha}} .
$$

Especially if one can neglect $\dot{H}$, it follows

$$
\tilde{p} \sim w \tilde{\rho}-(1+w) \frac{3 H^{\alpha}}{\kappa^{2} r_{c}^{2-\alpha}} .
$$

This shows that brane-world scenario may also suggest various forms of inhomogeneous modification for effective EOS of matter on the brane.
[1] R. R. Caldwell, M. Kamionkowski and N. N. Weinberg, Phys. Rev. Lett. 91, $071301 \quad$ (2003) arXiv:astro-ph/0302506; B. McInnes, JHEP 0208, $029 \quad$ (2002) arXiv:hep-th/0112066; hep-th/0502209 V. Faraoni, Int. J. Mod. Phys. D 11, 471 (2002); A. E. Schulz, Martin J. White, Phys. Rev. D 64, 043514 (2001);

S. Nojiri and S. D. Odintsov, Phys. Lett. B 562, 147 (2003) arXiv:hep-th/0303117; Phys. Lett. B 571, 1 (2003) arXiv:hep-th/0306212;

P. Singh, M. Sami and N. Dadhich, arXiv:hep-th/0305110

P. Gonzalez-Diaz, Phys. Lett. B586, 1 (2004) arXiv:astro-ph/0312579; hep-th/0408225

H. Stefancic, Eur. Phys. J. C 36, 523 (2004) arXiv:astro-ph/0312484;

M. Sami and A. Toporensky, Mod. Phys. Lett. A19, 1509 (2004) arXiv:gr-qc/0312009;

X. Meng and P. Wang, arXiv:hep-ph/0311070

Z. Guo, Y. Piao and Y. Zhang, arXiv:astro-ph/0404225

S. M. Carroll, A. De Felice and M. Trodden, arXiv:astro-ph/0408081

C. Csaki, N. Kaloper and J. Terning, arXiv:astro-ph/0409596

S. Tsujikawa and M. Sami, arXiv:hep-th/0409212

P. Gonzales-Diaz and C. Siguenza, Nucl. Phys. B697, 363 (2004) arXiv:astro-ph/0407421;

L. P. Chimento and R. Lazkoz, Mod. Phys. Lett. A19, 2479 (2004) arXiv:gr-qc/0405020; gr-qc/0307111

J. Hao and X. Li, arXiv:astro-ph/0404154

G. Calcagni, Phys. Rev. D 71, 023511 (2005) arXiv:gr-qc/0410027;

P. Wu and H. Yu, arXiv:astro-ph/0407424

J. Lima and J. S. Alcaniz, arXiv:astro-ph/0402265

S. Nesseris and L. Perivolaropoulos, Phys. Rev. D 70, 123529 (2004) arXiv:astro-ph/0410309;

M. Bento, O. Bertolami, N. Santos and A. Sen, arXiv:astro-ph/0412638

P. Scherrer, arXiv:astro-ph/0410508

Z. Guo, Y. Piao, X. Zhang and Y. Zhang, Phys. Lett. B 608177 (2005) arXiv:astro-ph/0410654;

[2] E. Elizalde, S. Nojiri and S. D. Odintsov, Phys. Rev. D 70, 043539 (2004) arXiv:hep-th/0405034;

E. Babichev, V. Dokuchaev and Yu. Eroshenko, arXiv:astro-ph/0407190

S. Sushkov, arXiv:gr-qc/0502084

K. Bronnikov, arXiv:gr-qc/0410119
L. Perivolaropoulos, arXiv:astro-ph/0412308

A. Vikman, Phys. Rev. D 71, $023515 \quad$ (2005) arXiv:astro-ph/0407107;

X. Zhang, H. Li, Y. Piao and X. Zhang, arXiv:astro-ph/ 0501652

M. Bouhmadi-Lopez and J. Jimenez-Madrid, arXiv:astro-ph/0404540

Y. Wei, arXiv:gr-qc/0410050 gr-qc/0502077

S. K. Srivastava, arXiv:hep-th/0411630

V. K. Onemli and R. Woodard, arXiv:gr-qc/0406098

M. Dabrowski and T. Stachowiak, arXiv:hep-th/0411199

I. Ya. Arefeva, A. S. Koshelev and S. Yu. Vernov, arXiv:astro-ph/0412638

E. Elizalde, S. Nojiri, S. D. Odintsov and P. Wang, Phys. Rev. D 71, 103504 (2005) arXiv:hep-th/0502082;

V. Sahni, arXiv:astro-ph/0502032

H. Wei, R.-G. Cai and D. Zeng, arXiv:hep-th/0501160

R. Curbelo, T. Gonzalez and I. Quiros, arXiv:astro-ph/0502141

B. Gumjudpai, T. Naskar, M. Sami and S. Tsujikawa, arXiv:hep-th/0502191

F. Lobo, arXiv:gr-qc/0502099

R. Lazkoz, S. Nesseris and L. Perivolaropoulos, arXiv:astro-ph/0503230

H. Lu, Z. Huang and W. Fang, arXiv:hep-th/0504038

X. Zhang, arXiv:astro-ph/0501160

F. Bauer, arXiv:gr-qc/0501078

A. Anisimov, E. Babichev, A. Vikman, arXiv:astro-ph/0504560

J. Sola and H. Stefancic, arXiv:astro-ph/0505133

A. Andrianov, F. Cannata and A. Kamenshchik, arXiv:gr-qc/0505087

[3] S. Nojiri and S. D. Odintsov, Phys. Rev. D 70, 103522 (2004) arXiv:hep-th/0408170.

[4] S. Nojiri, S. D. Odintsov and S. Tsujikawa, Phys. Rev. D 71, 063004 (2005) arXiv:hep-th/0501025.

[5] R. Caldwell, Phys. Lett. B 545, 23 (2002).

[6] J. D. Barrow, Class. Quant. Grav. 21, L79 (2004) arXiv:gr-qc/0403084;

S. Nojiri, S. D. Odintsov, Phys. Lett. B 595, 1 (2004), arXiv:hep-th/0405078;

J. D. Barrow, Class. Quant. Grav. 21, 5619 (2004) arXiv:gr-qc/0409062;

M. C. B. Abdalla, S. Nojiri and S. D. Odintsov, Class. Quant. Grav. 22, L35 (2005), arXiv:hep-th/0409177; S. Cotsakis and I. Klaoudatou, arXiv:gr-qc/0409022 V. Sahni and Yu. Shtanov, JCAP 0311, 014 (2003) arXiv:astro-ph/0202346; 
K. Lake, Class. Quant. Grav. 21, L129 (2004) arXiv:gr-qc/0407107;

M. Dabrowski, arXiv:gr-qc/0410033

L. Fernandez-Jambrina and R. Lazkoz, Phys. Rev. D 70, 121503 (2004) arXiv:gr-qc/0410124;

J. D. Barrow and C. Tsagas, arXiv:gr-qc/0411045

[7] S. Nojiri and S. D. Odintsov, Phys. Lett. B 599, 137 (2004) arXiv:astro-ph/0403622;

G. Allemandi, A. Borowiec, M. Francaviglia and S. D. Odintsov, arXiv:gr-qc/0504057

[8] S. Nojiri, S. D. Odintsov and M. Sasaki, arXiv:hep-th/0504052

M. Sami, A. Toporensky, P. Trejakov and S. Tsujikawa, arXiv:hep-th/0504154

[9] I. Brevik and O. Gorbunova, arXiv:gr-qc/0504001

[10] I. Brevik, arXiv:gr-qc/0404095

O. Gron, Astrophys. Space Sci. 173, 191 (1990);

S. Weinberg, Gravitation and Cosmology, John Wiley\& Sons, 1972.

[11] J. D. Barrow, Phys. Lett. B 180, 335 (1986); Nucl. Phys. B 310, 743 (1988).

[12] M. Szydlowski, W. Godlowski and R. Wojtak, arXiv:astro-ph/0505202
[13] H. Štefančić, arXiv:astro-ph/0504518

[14] H. Štefančić, arXiv:astro-ph/0411630

[15] L. Amendola, Phys. Rev. D62, 043511 (2000);

W. Zimdahl, D. Pavon and L. P. Chimento, Phys. Lett. B 521, 133 (2001);

G. Mangano, G. Miele and V. Pettorino, Mod. Phys. Lett. A 18, 831(2003);

G. Farrar and P. J. E. Peebles, Astrophys. J. 604, 1 (2004);

S. del Campo, R. Herrera and D. Pavon, Phys. Rev. D 70, 043540 (2004);

R.-G. Cai and A. Wang, JCAP 0503, 002 (2005);

D. Pavon and W. Zimdahl, arXiv:gr-qc/0505020

L. Chimento and D. Pavon, gr-qc/0505096

[16] M. Giovannini, arXiv:gr-qc/0504132 arXiv:astro-ph/0504655

[17] I. Brevik, S. Nojiri, S. D. Odintsov, L. Vanzo, Phys. Rev. D 70, 043520 (2004) arXiv:hep-th/0401073.

[18] C. Deffayet, G. Dvali and G. Gabadadze, Phys. Rev. D 65, 044023 arXiv:astro-ph/0105068.

[19] G. Dvali and M. S. Turner, arXiv:astro-ph/0301510 